\title{
Influence of Helicobacter pylori status and eradication on the serum levels of trefoil factors and pepsinogen test: serum trefoil factor 3 is a stable biomarker
}

\author{
Mitsuru Kaise $\cdot$ Jun Miwa $\cdot$ Ai Fujimoto $\cdot$ \\ Jun Tashiro • Daisuke Tagami • Hiromi Sano • \\ Yasukazu Ohmoto
}

Received: 19 December 2011/Accepted: 31 July 2012/Published online: 21 August 2012

(C) The International Gastric Cancer Association and The Japanese Gastric Cancer Association 2012

\begin{abstract}
Background and aim Emerging data indicate that serum trefoil factors (TFFs), especially TFF3, could be potential biomarkers for gastric cancer risk. We aimed to evaluate the influence of Helicobacter pylori (H. pylori) status and eradication on serum TFFs and the pepsinogen test.

Methods Healthy individuals who underwent a thorough medical checkup were enrolled in study 1 , and gastric ulcer patients who undertook $H$. pylori eradication therapy were enrolled in studies 2 and 3 . Serum levels of the TFFs (TFF1, TFF2 and TFF3), H. pylori antibody and pepsinogen test were examined in all studies. In study 3, TFF expressions in biopsy samples of the gastric mucosa were additionally examined before and 2 months after eradication.

Results In 1,260 healthy individuals enrolled in study 1, serum TFF1 and TFF2 levels were markedly different between $H$. pylori antibody-positive and -negative participants $(P<0.0001)$. Differences in serum TFF3 levels between $H$. pylori antibody-positive $(5.85 \pm 3.93 \mathrm{ng} / \mathrm{ml})$ and -negative subjects $(5.27 \pm 2.38 \mathrm{ng} / \mathrm{ml}) \quad$ were
\end{abstract}

M. Kaise $(\varangle) \cdot$ A. Fujimoto

Department of Gastroenterology, Toranomon Hospital,

2-2-2 Toranomon, Minato-ku, Tokyo 105-8470, Japan

e-mail: kaise@toranomon.gr.jp

J. Miwa $\cdot$ J. Tashiro $\cdot$ D. Tagami

Department of Gastroenterology, Toshiba General Hospital,

Tokyo, Japan

H. Sano

Department of Surgery, Nomura Hospital, Tokyo, Japan

Y. Ohmoto

Institute of Biomedical Innovation, Otsuka Pharmaceutical Co.,

Ltd, Tokushima, Japan statistically significant $(P=0.002)$ but small in absolute value. In 178 gastric ulcer patients enrolled in study 2, serum TFF1, TFF2 and positive rates of the pepsinogen test significantly decreased 2 months after $H$. pylori eradication therapy $(P<0.001)$. In contrast, serum TFF3 levels and positive rates of high TFF3 levels $(\geq 7 \mathrm{ng} / \mathrm{ml})$ did not significantly change with $H$. pylori-eradication until 5 years after eradication. In 18 gastric ulcer patients (study 3 ), TFF1 and TFF2 were mainly expressed in the foveolar epithelium, and TFF2 was additionally expressed in the pyloric glands. These expressions significantly decreased with H. pylori eradication. TFF3s were scarcely expressed in the gastric mucosa except in goblet cells of intestinal metaplasia, which did not change with $H$. pylori eradication.

Conclusion In serum TFFs and pepsinogen tests, only serum TFF3s were not significantly affected by $H$. pylori eradication, suggesting that serum TFF3 could be a stable biomarker of gastric cancer risk even after H.pylori eradication in contrast with the pepsinogen test.

Keywords Trefoil factor - Cancer biomarker . Helicobacter pylori - Gastric ulcer · Pepsinogen test

\section{Introduction}

The trefoil factor (TFF) family consists of three small polypeptides that are highly expressed in tissues containing mucus-producing cells. They play a key role in the maintenance of mucosal integrity [1] and oncogenic transformation, growth and metastatic extension of solid tumors [2-4]. TFFs, secreted mainly into the mucous layer, are also detected in the blood at much lower concentrations. Analyzing blood TFFs with an enzyme-linked 
immunosorbent assay (ELISA), we were the first to demonstrate that serum levels of TFF1, TFF2 and TFF3 were significantly higher in gastric cancer patients than in noncancerous controls [5]. The area under the ROC curve for predicting the presence of gastric cancer was significantly higher for a combination of the serum TFF3 value and pepsinogen (PG) test $(0.883)$ than for the PG test alone (0.823), the latter having been introduced as a populationbased screening tool for gastric cancer risk [6-8]. Therefore, the serum TFF3 value combined with the PG test might be a more valid biomarker for gastric cancer detection. Recently, the other group also demonstrated that tests for serum levels of TFF3 could improve gastric cancer screening [9].

Previous studies have also shown that the blood TFF3 value could be a biomarker for cancer detection in organs other than the stomach. In poorly differentiated endometrioid endometrial carcinoma (G3-EEC), TFF3 was highly expressed at the gene and protein level, and serum TFF3 could be a marker for the early detection and/or monitoring of G3-EEC patients [10]. In prostate cancer, patients with advanced disease had significantly higher plasma concentrations of TFF1, TFF2 and TFF3 compared to patients with localized disease. Differentiating between patients with localized and advanced disease using a cutoff level for plasma TFF3 had sensitivity and specificity of 74 and $81 \%$, respectively; thus, plasma TFF3 could be a marker for differentiating advanced cancers from localized disease [11].

These data suggest that blood TFFs, especially TFF3, could be potential cancer biomarkers, but basic data are necessary to evaluate whether clinical factors other than cancer can affect the status of TFF-containing cells and alter blood levels of TFFs. In the present study, we aimed mainly to elucidate the influence of Helicobacter pylori status and eradication on serum levels of TFFs.

\section{Materials and methods}

This study was approved by the Ethics Committees of Toshiba General Hospital and Nomura Hospital, and was conducted in accordance with the Declaration of Helsinki (as revised in 1989).

In study 1 , individuals who had a medical health checkup scheduled at the Health Medical Center of Nomura Hospital in 2008 and 2009 were eligible. Those who provided written informed consent and underwent upper gastrointestinal endoscopy or an upper gastrointestinal series were prospectively included. The following exclusion criteria applied: (1) upper gastrointestinal neoplasia or peptic ulcer (opened or scarred) diagnosed by upper gastrointestinal endoscopy or an upper gastrointestinal series;
(2) previous gastrointestinal surgery; (3) ongoing treatment for cancer of organs other than the upper gastrointestinal tract; (4) severe comorbidities, including hepatic, renal, cardiopulmonary and hematologic disease.

In study 2 , we prospectively recruited $H$. pylori-positive gastric ulcer patients who underwent $H$. pylori eradication therapy between 2006 and 2010 at Toshiba General Hospital with written informed consent.

Fasting serum was collected from the healthy participants on the day of their medical health checkup and from gastric ulcer patients before and 2 months after H. pylori eradication treatment. We asked successfully eradicated gastric ulcer patients to undergo endoscopy and serum sampling 1 and 5 years after successful eradication of H. pylori, and some of the patients agreed to undergo the follow-up examinations. The samples were stored at $-80{ }^{\circ} \mathrm{C}$ until analysis. In the healthy participants, H. pylori status was evaluated by detection of specific $H$. pylori $\operatorname{IgG}$ antibodies using a commercial enzyme immunoassay kit (E plate; Eiken Kagaku, Tokyo, Japan). In gastric ulcer patients, the diagnosis of current $H$. pylori infection was made on the basis of the histology, rapid urease test and/or ${ }^{13} \mathrm{C}$ urea breath test. A triple therapy for $H$. pylori eradication [amoxicillin $750 \mathrm{mg}$ twice a day, clarithromycin $200 \mathrm{mg}$ twice a day, and a proton pump inhibitor (omeprazole $20 \mathrm{mg}$ or lansoprazole $30 \mathrm{mg}$ or rabeprazole $10 \mathrm{mg}$ ) twice a day for 1 week] was performed after ulcer healing was endoscopically observed. Successful H. pylori eradication was defined as being achieved if all three tests (histology, rapid urease test and ${ }^{13} \mathrm{C}$ urea breath test) were H. pylori negative.

Serum levels of TFFs were analyzed by ELISA as previously reported [5]. In brief, human TFF1, TFF2 and TFF3 expression plasmids were constructed from Marathon-Ready ${ }^{\mathrm{TM}}$ cDNA Human Small Intestine and Stomach (Clontech Laboratories, Inc., Mountain View, CA, USA). Using purified polyclonal antibodies prepared from rabbits immunized with recombinant human TFFs, ELISAs for human TFFs were constructed. Concentrations of human TFFs in the samples were calculated from a standard curve constructed from recombinant human TFFs. The assay sensitivities for TFF1, TFF2 and TFF3 were 7, 30 and $30 \mathrm{pg} / \mathrm{ml}$, respectively.

Serum PG I and PG II were also measured by chemiluminescent enzyme immunoassay using commercial kits (Lumipulse pepsinogen I \& II; Fujirebio, Inc., Tokyo, Japan). Serum PG status was defined as positive when the criteria of both serum PG I level $\leq 70 \mathrm{ng} / \mathrm{ml}$ and PG I/II ratio $\leq 3.0$ were simultaneously fulfilled.

In study 3 , we prospectively recruited $H$. pylori-positive gastric ulcer patients who underwent $H$. pylori eradication therapy between 2006 and 2010 at Toshiba General Hospital with written informed consent. Fasting serum was 
collected before and 2 months after $H$. pylori eradication treatment. Endoscopy was done before and after eradication, and two biopsy samples of the gastric mucosa were obtained from the upper corpus and antrum of the great curvature in each subject. To evaluate the relationship between serum TFF levels and tissue expression of TFFs in the stomach, immunohistochemical examination was performed using biopsied samples as reported before [5]. Tissue expression levels of TFFs were scored as negative (0), mildly (1), moderately (2) and strongly positive (3).

We examined relationships between the serum levels of TFFs and Helicobacter pylori status and clinical factors including age, gender, PG test or gastric ulcer presence. Continuous variables were compared using $t$ tests. A twosided $P$ value $<0.05$ was considered statistically significant. All analyses were performed using STATA 11.0 software (STATA Co., College Station, TX, USA). Quantitative data are summarized as mean \pm standard deviation.

\section{Results}

Serum TFFs in healthy individuals

In study $1,1,303$ healthy individuals were eligible for this study; 43 met the exclusion criteria, with the remaining 1,260 individuals (age $52.5 \pm 12.4$ years) finally enrolled as healthy participants.

Clinical factors that might affect serum levels of TFFs were evaluated. At first, correlations between age and serum TFFs were analyzed. The correlation coefficient $(r)$ and coefficient of determination $\left(r^{2}\right)$ between age and serum TFF1 were 0.3554 and 0.1263 , respectively, meaning that a weak correlation between age and serum TFF1 existed (Fig. 1). Similarly, a weak correlation also existed between age and serum TFF2 $\left(r=0.3494, r^{2}=0.1221\right.$; Fig. 1). In contrast, however, no significant correlation between age and serum TFF3 existed $(r=0.1410$, $r^{2}=0.0199$; Fig. 1). These relationships were also tested by stratifying the individuals according to being $H$. pylori antibody positive or negative. Serum TFF3 levels were not correlated with age in either $H$. pylori antibody-positive or -negative individuals. Serum TFF1 and TFF2 levels were also correlated with age in $H$. pylori antibody-negative individuals, but were not in $H$. pylori antibody-positive ones (Fig. 2).

The enrolled healthy participants consisted of 715 males and 545 females. The mean age of the males (53.7 \pm 12.7 years) was 3 years older than the females $(50.7 \pm$ 12.0 years). Serum TFF1 levels of males and females were similar at $1.14 \pm 1.35$ and $1.06 \pm 1.35 \mathrm{ng} / \mathrm{ml}$, respectively $(P=0.15)$. Serum TFF2 levels of males and females were also similar $(3.19 \pm 1.86$ and $3.29 \pm 1.95 \mathrm{ng} / \mathrm{ml}$, respectively, $P=0.83$ ), as were serum TFF3 levels of males and females $(5.39 \pm 2.08$ and $5.54 \pm 3.81 \mathrm{ng} / \mathrm{ml}$, respectively, $P=0.15)$. Taken together, gender did not affect serum levels of TFFs.

Of the 1,260 healthy individuals, 867 were $H$. pylori antibody negative and 393 were $H$. pylori antibody positive. Serum levels of TFF1 and TFF2 in H. pylori antibodypositive participants $(2.47 \pm 1.68$ and $4.81 \pm 2.38 \mathrm{ng} / \mathrm{ml}$, respectively) were significantly higher than in $H$. pylori antibody-negative individuals $(0.49 \pm 0.40$ and $2.52 \pm$ $1.02 \mathrm{ng} / \mathrm{ml}$, respectively; $P<0.001$; Fig. 3). Serum levels of TFF3 in $H$. pylori antibody-positive individuals (5.85 \pm $3.93 \mathrm{ng} / \mathrm{ml}$ ) and $H$. pylori antibody-negative individuals $(5.27 \pm 2.38 \mathrm{ng} / \mathrm{ml})$ were similar in absolute value, but the difference was statistically significant $(P=0.002)$.

Influence of $H$. pylori eradication therapy on the levels of serum TFFs and pepsinogen test

In study 2, 178 gastric ulcer patients were enrolled (age $62.1 \pm 8.3$ years, male/female ratio 151:27). In all patients, $H$. pylori eradication therapy was administered after gastric ulcers had been proven benign pathologically and demonstrated to be healed by endoscopy. In 163 of these 178 patients, $H$. pylori was successfully eradicated.

Before $H$. pylori eradication therapy, serum levels of TFF1, TFF2 and TFF3 in gastric ulcer patients were
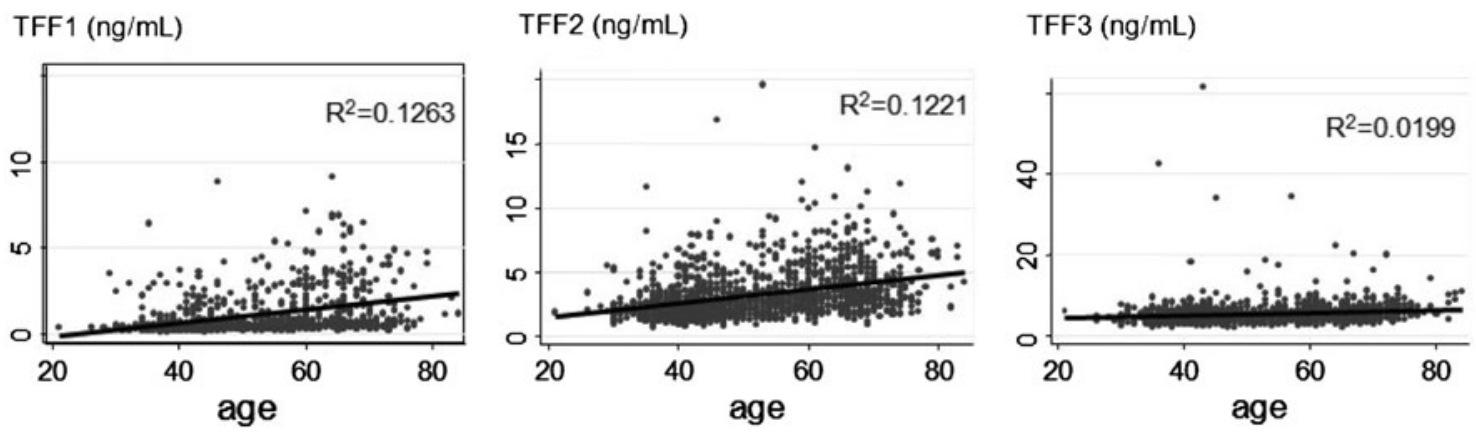

Fig. 1 Correlations between serum trefoil factors (TFFs) and age 
H. pylori -negative controls $(\mathrm{n}=867)$
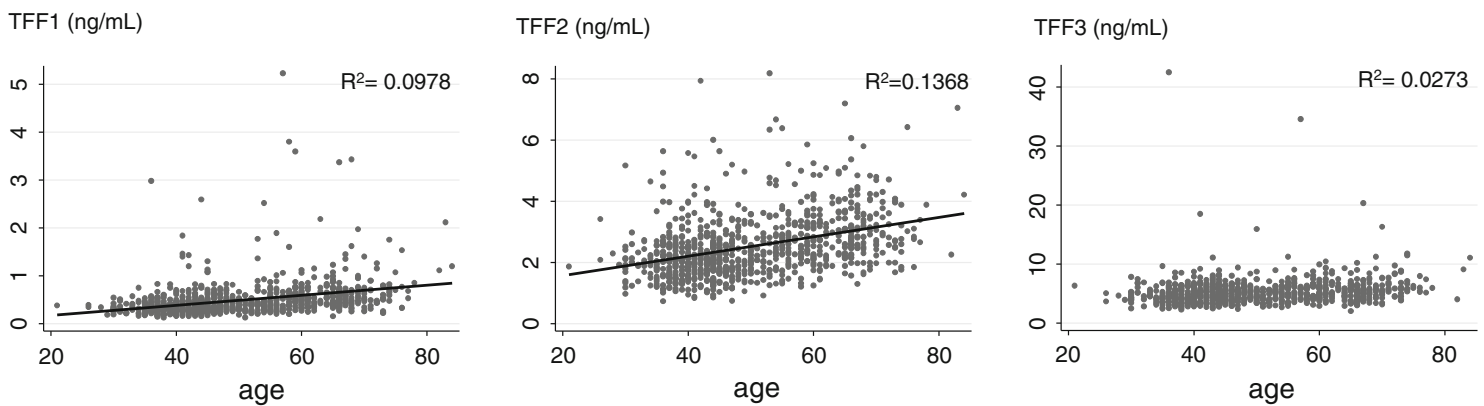

H. pylori -positive controls $(n=393)$
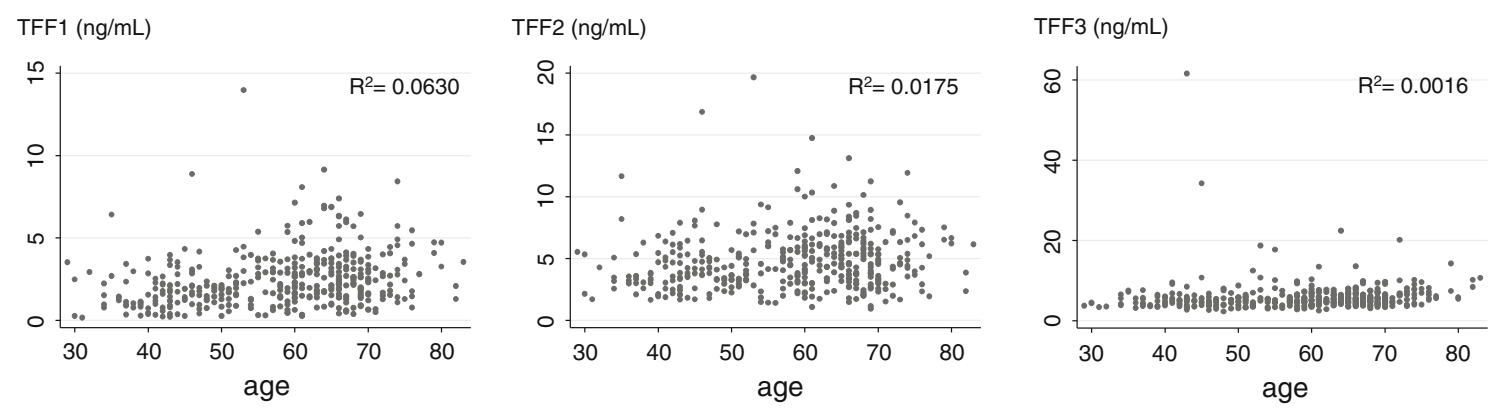

Fig. 2 Correlations between serum trefoil factors (TFFs) and age. H. pylori antibody-positive and -negative subjects

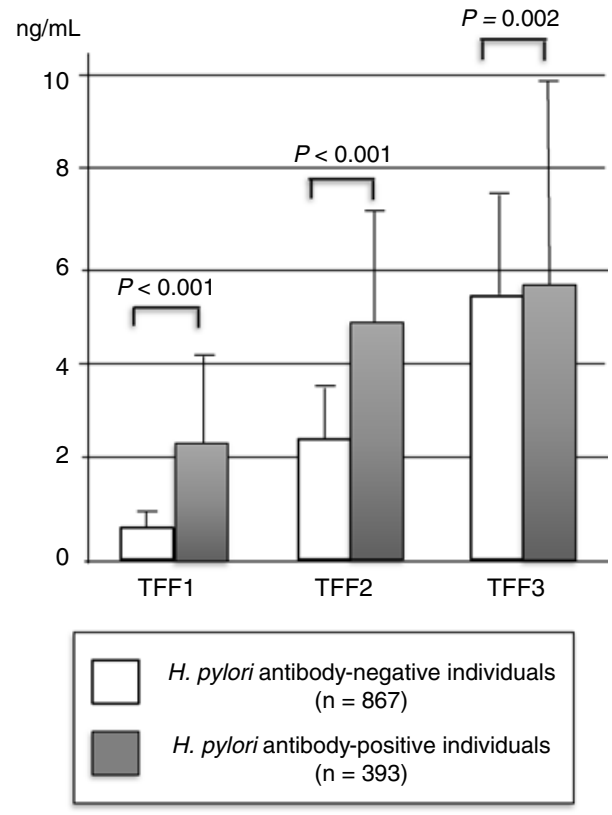

Fig. 3 Comparison of serum levels of trefoil factors (TFFs), expressed as $\mathrm{ng} / \mathrm{ml}$, in Helicobacter pylori antibody-positive $(n=393)$ and $H$. pylori antibody-negative $(n=867)$ healthy participants. Results are shown as mean \pm SD

$3.08 \pm 1.50, \quad 6.95 \pm 2.86, \quad$ and $\quad 7.51 \pm 2.59 \mathrm{ng} / \mathrm{ml}$, respectively. Two months after $H$. pylori eradication therapy, serum levels of TFF1 and TFF2 significantly decreased to $1.22 \pm 0.60$ and $4.29 \pm 1.49 \mathrm{ng} / \mathrm{ml}$, respectively $(P<0.001)$. In contrast, serum levels of TFF3 did not change significantly 2 months after $H$. pylori eradication therapy $(7.11 \pm 1.89 \mathrm{ng} / \mathrm{ml} ; P=0.07)$. We analyzed the data by dividing the gastric ulcer patients into two groups: those in whom $H$. pylori eradication was successful $(n=163)$ and those in whom it was unsuccessful $(n=15)$. In the successfully treated patients, the effect of H. pylori eradication therapy on serum levels of TFFs was similar to all the gastric ulcer patients, including the unsuccessfully treated patients (Fig. 4). In contrast, the effect of the eradication therapy on serum TFF levels was different in the unsuccessfully treated patients. Serum levels of TFF1, TFF2 and TFF3 did not change before and after eradication therapy (Fig. 4). Our previous study showed that serum TFF3 concentrations $\geq 7 \mathrm{ng} / \mathrm{ml}$ could be a positive cutoff criterion for predicting gastric cancer presence. We tested whether positive rates of serum TFF3 levels could be decreased by $H$. pylori eradication. In both $H$. pylori eradicated and un-eradicated patients, the positive rates of serum TFF3 levels did not significantly change with eradication therapy (Table 1). The evaluations of serum TFF3 levels changed from positive to negative in $38.4 \%$ of successfully eradicated patients whose preeradication TFF3 levels were $\geq 7 \mathrm{ng} / \mathrm{ml}$. The conversion from positive to negative was observed in $28.6 \%$ of unsuccessfully eradicated patients whose pre-eradication TFF3 levels were $\geq 7 \mathrm{ng} / \mathrm{ml}$. 
Fig. 4 Effect of Helicobacter pylori eradication therapy on serum levels of trefoil factors (TFFs), expressed as $\mathrm{ng} / \mathrm{ml}$, in gastric ulcer patients successfully $(n=163)$ and unsuccessfully ( $n=15)$ cured of H. pylori. Two months after H. pylori eradication therapy, serum levels of TFF1 and TFF2 had significantly decreased but serum TFF3 levels had not changed in patients successfully cured of $H$. pylori. In patients in whom $H$. pylori eradication was unsuccessful, serum TFF levels were not altered by $H$. pylori eradication therapy
$H$. pylori eradication successful
\[ (\mathrm{n}=163) \]

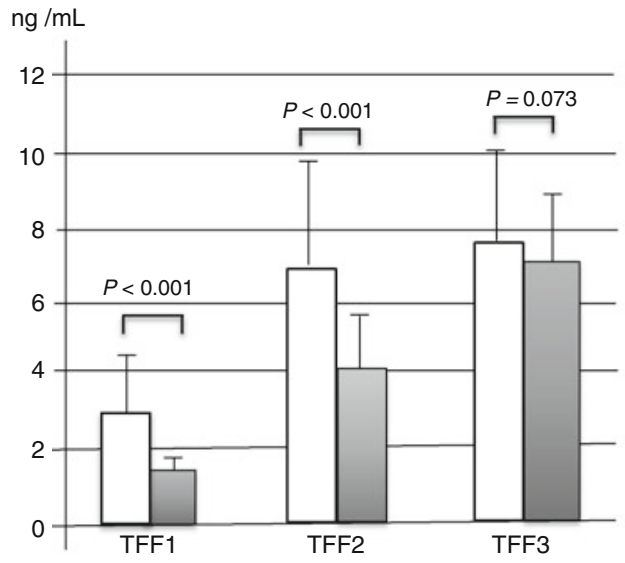

$H$. pylori eradication unsuccessful $(n=15)$

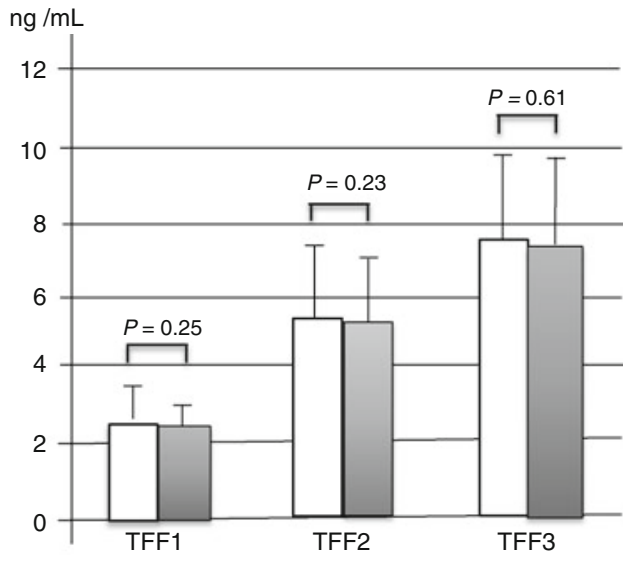

$\square$ before eradication therapy

after eradication therapy

Table 1 Influence of Helicobacter pylori eradication therapy on the pepsinogen (PG) test and positive rates of high serum TFF3 levels ( $\geq 7$ ng/ $\mathrm{ml}$ ) in gastric ulcer patients

\begin{tabular}{|c|c|c|c|c|c|}
\hline & PG I (ng/ml) & PG II (ng/ml) & PG I/II ratio & $\begin{array}{l}\text { Positive PG } \\
\text { test }(\%)\end{array}$ & $\begin{array}{l}\text { Positive rate of high } \\
\text { serum TFF3 }(\geq 7 \mathrm{ng} / \mathrm{ml})(\%)\end{array}$ \\
\hline \multicolumn{6}{|c|}{ H. pylori eradication successful $(n=163)$} \\
\hline Before eradication therapy & $76.4 \pm 56.6$ & $26.8 \pm 13.2$ & $2.77 \pm 1.01$ & 48.7 & 53.8 \\
\hline After eradication therapy & $40.5 \pm 19.1$ & $8.4 \pm 3.4$ & $4.85 \pm 3.38$ & 44.8 & 44.8 \\
\hline$P$ value & $<0.0001$ & $<0.0001$ & $<0.0001$ & $<0.0001$ & 0.16 \\
\hline \multicolumn{6}{|c|}{ H. pylori eradication unsuccessful $(n=15)$} \\
\hline Before eradication therapy & $74.7 \pm 62.4$ & $26.4 \pm 12.7$ & $2.83 \pm 1.08$ & 53.3 & 46.7 \\
\hline After eradication therapy & $86.2 \pm 98.7$ & $27.1 \pm 23.8$ & $3.21 \pm 1.24$ & 40.0 & 33.3 \\
\hline$P$ value & 0.71 & 0.92 & 0.38 & 0.72 & 0.71 \\
\hline
\end{tabular}

Values of PG I, PG II and PG I/II ratio are mean \pm SD

To determine the long-term effect of successful $H$. pylori eradication, 20 gastric ulcer patients underwent additional serum sampling 1 and 5 years after eradication therapy. Serum TFF3 levels did not change until 5 years after eradication (Fig. 5). Positive rates of serum TFF3 levels $(\geq 7 \mathrm{ng} / \mathrm{ml})$ also did not alter until 5 years after eradication; the rates before eradication and 2 months, 1 year and 5 years after eradication were 50,40, 45 and $50 \%$, respectively. These data indicated that serum TFF3 levels were stably sustained at least 5 years after $H$. pylori eradication. In contrast, serum TFF1 levels decreased 2 months after eradication, and this was maintained as low for 5 years after eradication. Serum TFF1 levels decreased 2 months after eradication and additionally decreased 5 years after eradication therapy.

Next, we tested whether $H$. pylori eradication affected the PG test, which is used as a predictor for gastric cancer presence in population-based screening examinations. In patients in whom $H$. pylori was successfully eradicated
( $n=163$ ), PG I and PG II significantly decreased after eradication therapy (Table 1). Because the decrease in PG II was larger than in PG I, the PG I/II ratio significantly increased. The rate of positive PG tests, used as a criterion for predicting gastric cancer risk, significantly decreased from 48.7 to $11.3 \%$ after successful $H$. pylori eradication. The PG test results changed from positive to negative in $87.1 \%$ of successfully eradicated patients whose PG test before eradication was positive. In contrast, the conversion from positive to negative was observed in $25 \%$ of unsuccessfully eradicated patients whose PG test before the therapy was positive.

The correlation between histology and serum TFF levels before and after $H$. pylori eradication

In study 3 , we prospectively recruited $18 \mathrm{H}$. pylori-positive gastric ulcer patients who underwent $H$. pylori eradication 


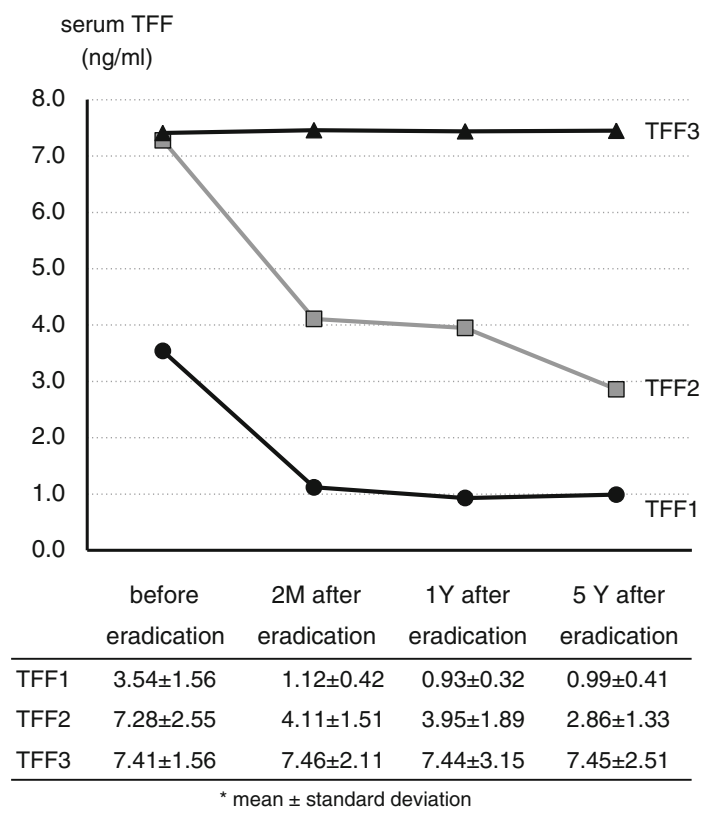

Fig. 5 A long-term effect of successful $H$. pylori eradication on serum levels of trefoil factors (TFFs) in gastric ulcer patients successfully cured of $H$. pylori $(n=20)$

therapy and examined serum TFF levels as well as tissue expression of TFFs before and 2 months after eradication. H. pylori was successfully eradicated in these 18 patients. Serum levels of TFF1 $(2.67 \pm 1.21 \mathrm{ng} / \mathrm{ml})$ and TFF2 $(5.96 \pm 2.51 \mathrm{ng} / \mathrm{ml})$ before eradication decreased significantly to $0.95 \pm 0.71$ and $3.33 \pm 1.81 \mathrm{ng} / \mathrm{ml}$, respectively, 2 months after eradication. Serum levels of TFF3 $(5.97 \pm 1.71 \mathrm{ng} / \mathrm{ml})$ before eradication were comparable to those $(6.15 \pm 1.15 \mathrm{ng} / \mathrm{ml}) 2$ months after eradication. These alterations of serum TFF were compatible with the data obtained in study 2 .

In these patients, we evaluated TFF expression in the gastric mucosa before and 2 month after $H$. pylori eradication. As shown in Fig. 6a, b, TFF1s were mainly expressed in the foveolar epithelium of the stomach. TFF2s were mainly expressed in the foveolar epithelium and pyloric glands. In contrast to TFF1s and TFF2s, TFF3s were exclusively expressed in goblet cells of intestinal metaplasia, but were scarcely expressed in non-metaplastic gastric mucosa. TFF1 expression scores in the gastric mucosa of the corpus and antrum were significantly decreased by $H$. pylori eradication (Table 2). TFF2 expression scores in the gastric antrum had a declining tendency in $H$. pylori eradication. TFF3 expressions in the biopsied gastric mucosa were very limited both before and after $H$. pylori eradication, and no remarkable alteration was found with $H$. eradication.

\section{Discussion}

PG testing has been introduced as a population-based screening tool for gastric cancer in Japan because it is simple and inexpensive [6-8]. However, cumulative data have shown some limitations, one of which is an insufficient predictive value of gastric cancer risk in $\mathrm{H}$. pylorieradicated individuals. A previous study reported that the evaluation of PG test results changed from positive to negative in $80.4 \%$ of PG test-positive cases following successful eradication [12]. In this study, the conversion rate was $87.1 \% 2$ months after eradication, indicating that PG testing may underestimate gastric cancer risks in H. pylori-eradicated individuals. In contrast to PG testing, $H$. pylori eradication seems to influence serum TFF3 levels in a different manner. $H$. pylori eradication did not significantly alter serum TFF3 levels in gastric ulcer patients until at least 5 years after eradication therapy. The evaluation of serum TFF3 levels changed from positive to negative in 38.4 and $28.6 \%$ of TFF3 test-positive cases after successful and unsuccessful $H$. pylori eradication, respectively. Taken together with our previous data, our results show that serum TFF3 could be a valid risk marker for gastric cancer even after $H$. pylori eradication.

The present study is the first to demonstrate that the $H$. pylori status influences serum levels of the TFF family in a different manner. Long-term infection with $H$. pylori in healthy individuals significantly increased serum TFF1, TFF2 and TFF3 levels. However, the difference in absolute value of serum TFF3 levels between H. pylori antibodypositive and -negative individuals was smaller than those of serum TFF1 and TFF2. H. pylori eradication significantly decreased serum TFF1 and TFF2, but did not decrease serum TFF3 levels. These differences in the H. pylori-related responses among TFF family members may be explained by the topographical divergence of expression among the TFF family; TFF1 is expressed in the gastric surface epithelial cells of the antrum and corpus, and TFF2 is expressed in the gastric surface epithelial cells and pyloric glands, whereas TFF3 expression is not observed in epithelial cells of the stomach and is restricted to intestinal goblet cells of metaplasia, as shown in the present and previous studies [13, 14]. H. pylori infection may directly affect the state of the gastric epithelia and change the levels of TFF1 and TFF2 expressed in the gastric epithelial cells. In contrast, $H$. pylori-related changes in serum TFF3 levels may be limited because they are expressed only in intestinal metaplasia.

Aging is a factor that can influence blood levels of members of the TFF family. In the healthy participants and gastric ulcer patients enrolled in our study, aging did not 
a

\section{Before Helicobacter pylori eradication (Antrum mucosa)}

\section{TFF1}

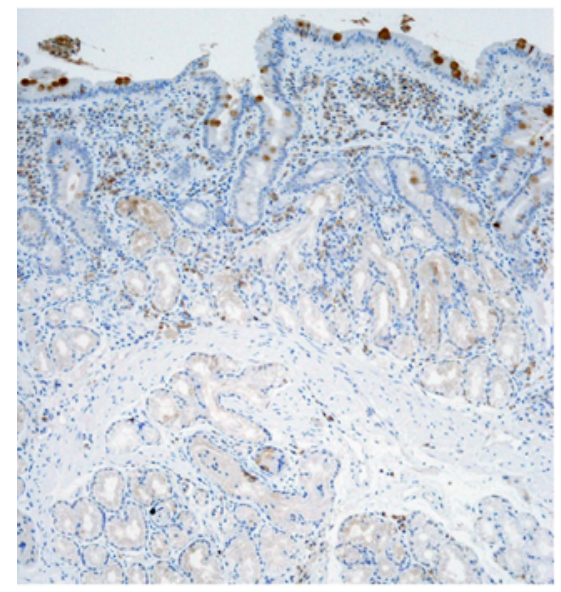

TFF2

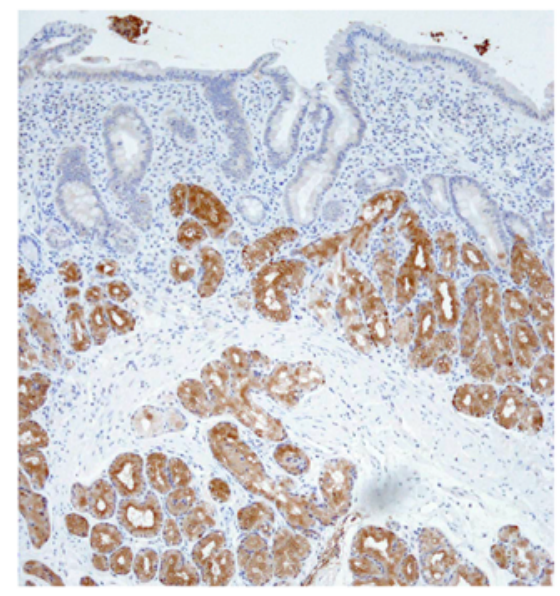

TFF3

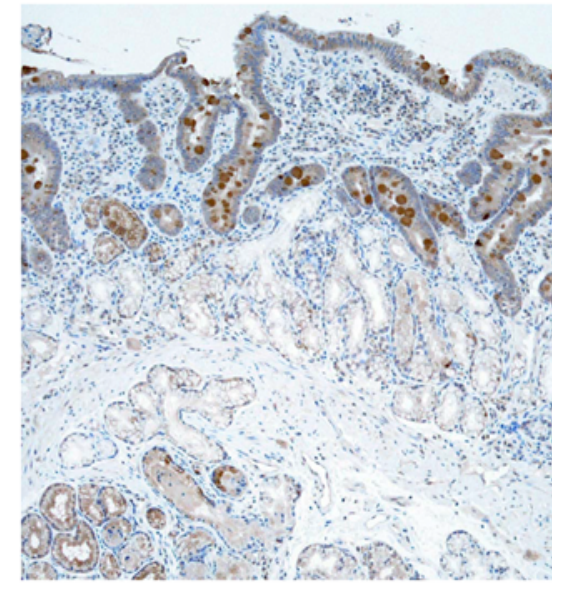

\section{After Helicobacter pylori eradication (Antrum mucosa)}

\section{TFF1}

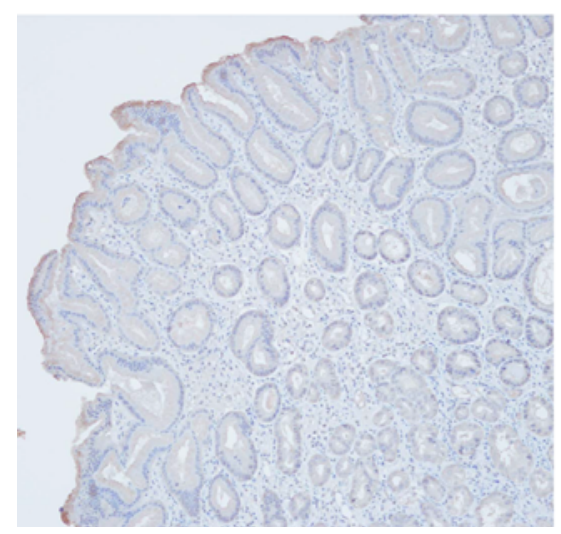

TFF2

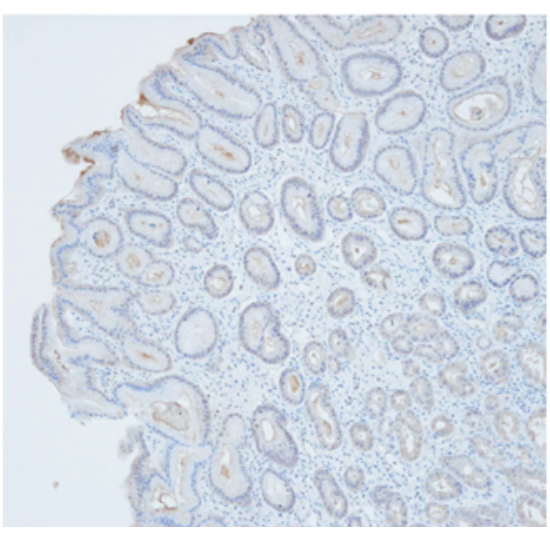

TFF3

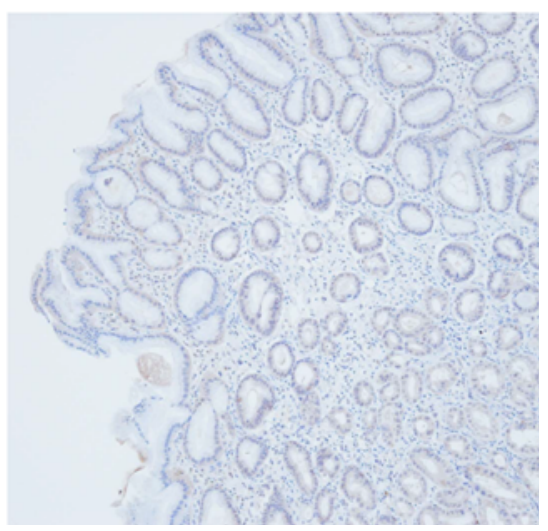

Fig. 6 Representative pictures of immunohistochemistry of trefoil factors in the mucosa of gastric antrum (a) and corpus (b). TFF1 and TFF2 were mainly expressed in the foveolar epithelium, and TFF2 was additionally expressed in the pyloric glands. These expressions significantly decreased with $H$. pylori eradication. TFF3s were scarcely expressed in the gastric mucosa except in goblet cells of intestinal metaplasia, which did not change with $H$. pylori eradication affect serum TFF3 levels, but was weakly correlated with serum TFF1 and TFF2 levels. In H. pylori-negative healthy subjects, aging-related increments of serum TFF1 and TFF2 were evident. In contrast, these increments were not observed in $H$. pylori-positive subjects, suggesting that H. pylori infection more strongly increases serum TFF1 and TFF2 levels as compared to aging. Gender might be a candidate for the modulation, but did not affect serum levels of TFF1, TFF2 or TFF3. The present study suggests that serum levels of TFFs in gastric ulcer patients seem to be relatively higher than those in healthy individuals. However, the ages of gastric ulcer patients were almost 10 years older than those of healthy individuals, and ages and genders were unmatched between these two groups. Thus, the difference in serum TFF levels is not conclusive, and further studies are required.

Our study has some limitations. The influence of $H$. pylori eradication on TFF serum levels was only examined in gastric ulcer patients. Therefore, it is unclear how H. pylori eradication affects serum TFF levels in subjects including gastric cancer patients. This is an important issue to examine in further studies to confirm that serum TFF3 is a valid risk marker even in the H. pylori-eradicated population with gastric cancer.

In conclusion, of serum TFF values and the pepsinogen test, only serum TFF3s were not considerably affected by 
TFF1

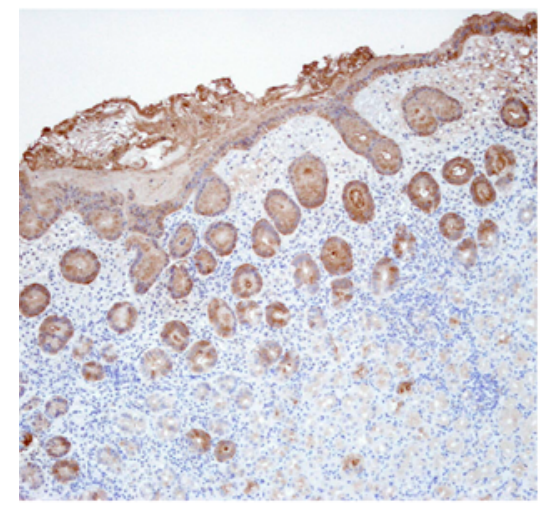

TFF2

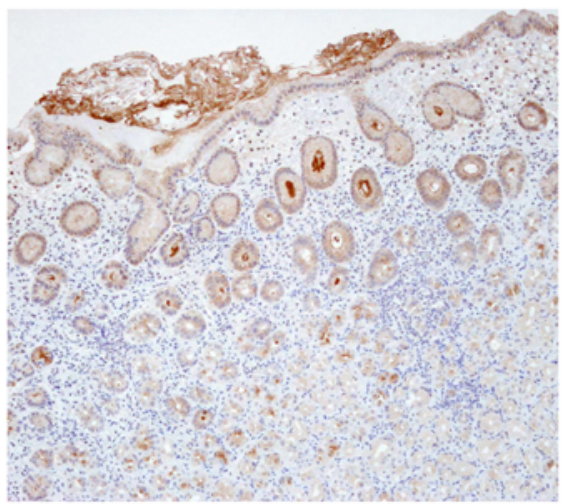

TFF3

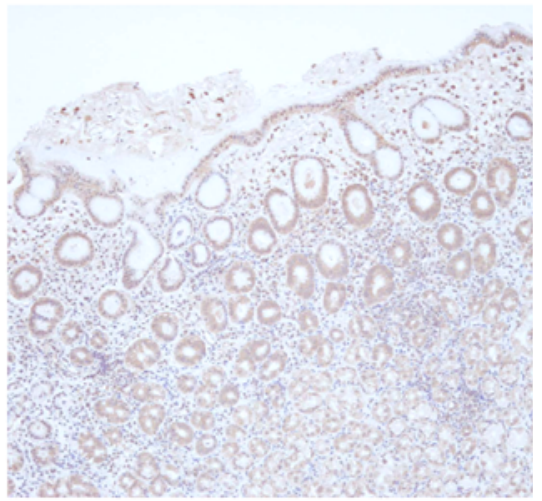

\section{After Helicobacter pylori eradication (Corpus mucosa)}
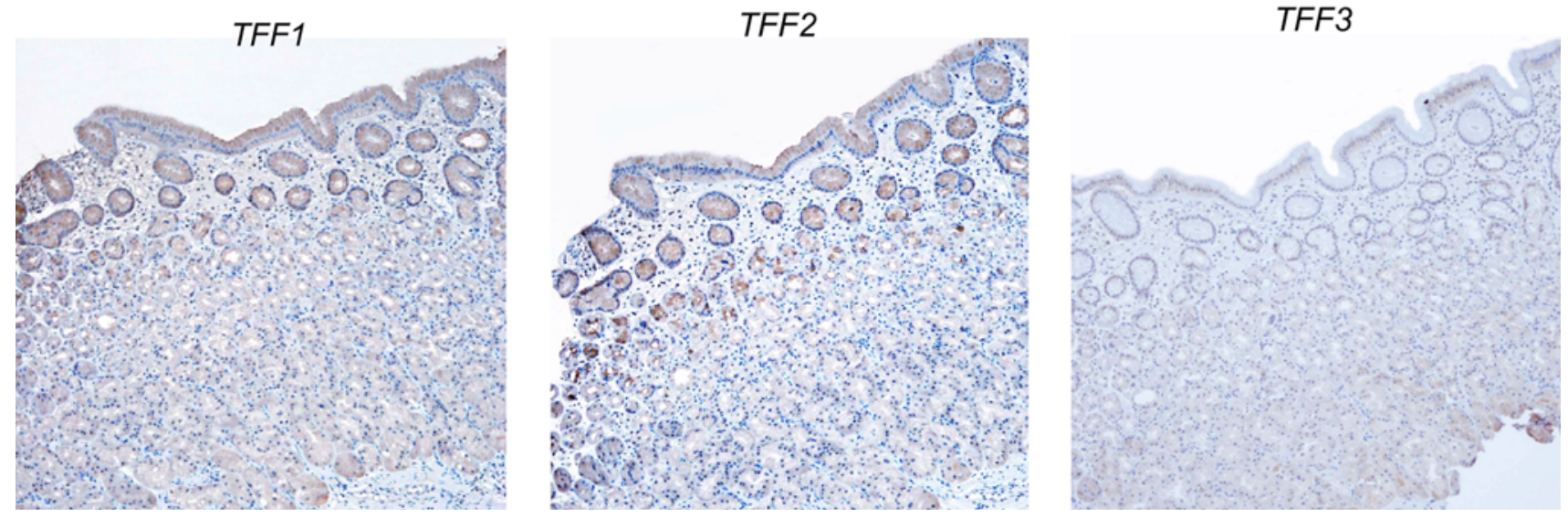

Fig. 6 continued

Table 2 Effect of Helicobacter pylori eradication therapy on trefoil factor (TFF) expressions in the gastric mucosa in gastric ulcer patients successfully cured of $H$. pylori $(n=18)$

\begin{tabular}{lccc}
\hline & Before eradication & $\begin{array}{l}2 \text { months after } \\
\text { eradication }\end{array}$ & $P$ value \\
\hline \multicolumn{2}{l}{ Corpus mucosa } & & \\
TFF1 & $2.56 \pm 0.62$ & $1.83 \pm 1.14$ & 0.02 \\
TFF2 & $1.20 \pm 0.65$ & $1.06 \pm 0.59$ & 0.496 \\
TFF3 & $0.06 \pm 0.24$ & $0.0 \pm 0.0$ & \\
Antrum mucosa & & \\
TFF1 & $2.528 \pm 0.776$ & $1.639 \pm 1.040$ & 0.005 \\
TFF2 & $0.611 \pm 0.631$ & $0.306 \pm 0.389$ & 0.081 \\
TFF3 & $0.056 \pm 0.236$ & $0.0 \pm 0.0$ & \\
\hline
\end{tabular}

H. pylori status, eradication and aging, suggesting that serum TFF3 could be a stable biomarker of gastric cancer risk even after $H$. pylori eradication.
Acknowledgments The authors would like to thank Ms. Keiko Hayakawa for her helpful assistance.

Conflict of interest None.

\section{References}

1. Taupin D, Podolsky DK. Trefoil factors: initiators of mucosal healing. Nat Rev Mol Cell Biol. 2003;4:721-32.

2. Peterson AJ, Menheniott TR, O'Connor L, Walduck AK, Fox JG, Kawakami K, et al. Helicobacter pylori infection promotes methylation and silencing of trefoil factor 2, leading to gastric tumor development in mice and humans. Gastroenterology. 2010;139:2005-17.

3. Dhar DK, Wang TC, Tabara H, Tonomoto Y, Maruyama R, Tachibana M, et al. Expression of trefoil factor family members correlates with patient prognosis and neoangiogenesis. Clin Cancer Res. 2005;11:6472-8. 
4. Perry JK, Kannan N, Grandison PM, Mitchell MD, Lobie PE. Are trefoil factors oncogenic? Trends Endocrinol Metab. 2008;19: 74-81.

5. Kaise M, Miwa J, Tashiro J, Ohmoto Y, Morimoto S, Kato M, et al. The combination of serum trefoil factor 3 and pepsinogen testing is a valid non-endoscopic biomarker for predicting the presence of gastric cancer -a new marker for gastric cancer risk. J Gastroenterol. 2011;46:736-45.

6. Miki K, Ichinose M, Kakei N, Yahagi N, Matsushima M, Tsukada $\mathrm{S}$, et al. The clinical application of the serum pepsinogen I and II levels as a mass screening method for gastric cancer. Adv Exp Med Biol. 1995;362:139-43.

7. Kitahara F, Kobayashi K, Sato T, Kojima Y, Araki T, Fujino MA, et al. Accuracy of screening for gastric cancer using serum pepsinogen concentrations. Gut. 1999;44:693-7.

8. Dinis-Ribeiro M, Yamaki G, Miki K, Costa-Pereira A, Matsukawa M, Kurihara M. Meta-analysis on the validity of pepsinogen test for gastric carcinoma, dysplasia or chronic atrophic gastritis screening. J Med Screen. 2004;11:141-7.

9. Aikou S, Ohmoto Y, Gunji T, Matsuhashi N, Ohtsu H, Miura H, et al. Tests for serum levels of trefoil factor family proteins can improve gastric cancer screening. Gastroenterology. 2011;141: 837-45

10. Bignotti E, Ravaggi A, Tassi RA, Calza S, Rossi E, Falchetti M, et al. Trefoil factor 3: a novel serum marker identified by gene expression profiling in high-grade endometrial carcinomas. Br J Cancer. 2008;99:768-73.

11. Vestergaard EM, Borre M, Poulsen SS, Nexø E, Tørring N. Plasma levels of trefoil factors are increased in patients with advanced prostate cancer. Clin Cancer Res. 2006;12:807-12.

12. Kawai T, Miki K, Ichinose M, et al. Changes in evaluation of the pepsinogen test result following Helicobacter pylori eradication therapy in Japan. Inflammopharmacology. 2007;15:31-5.

13. Leung WK, Yu J, Chan FK, To KF, Chan MW, Ebert MP, et al. Expression of trefoil peptides (TFF1, TFF2, and TFF3) in gastric carcinomas, intestinal metaplasia, and non-neoplastic gastric tissues. J Pathol. 2002;197:582-8.

14. Madsen J, Nielsen O, Tornoe I, Thim L, Holmskov U. Tissue localization of human trefoil factors 1,2 and 3. J Histochem Cytochem. 2007;55:505-13. 